\title{
Termes d'adresse et pulsion invocante chez Léonora Miano
}

\author{
Maribel PEŃALVER VICEA \\ Universidad de Alicante \\ mi.penalver@ua.es
}

\section{Resumen}

El presente artículo examina la presencia de la interpelación en L'intérieur de la nuit de Léonora Miano. Desde una perspectiva psicoanalítica y semánticopragmática, intentaremos demostrar la forma en que este mecanismo oral y discursivo construye la identidad del (inter)locutor, permitiendo a la vez mostrar la "pulsión invocante» (Lacan, 1973), reflejo del inconsciente.

Palabras clave: Identidad. Alteridad. Inconsciente. Freud. Lacan.

\section{Abstract}

This article examines the terms of address in L'intérieur de la nuit by Léonora Miano. Following a psychoanalytic and semantic-pragmatic perspective, I will try to show the way in which this discursive and oral marker constructs and models the identity of the speaker, as well as showing the "invocant impulse» (Lacan, 1973), reflection of the unconscious.

Key words: Identity. Otherness. Unconscious. Freud. Lacan.

\section{Résumé}

Cet article sera consacré aux termes d'adresse dans L'intérieur de la nuit de Léonora Miano. Suivant une approche psychanalytique et sémantico-pragmatique, cette contribution tentera de montrer la façon dont les termes d'adresse, en tant que marqueurs discursifs d'oralité, participent à la construction de l'identité, ainsi qu'à la monstration de la "pulsion invocante » (Lacan, 1973), miroir de l'inconscient.

Mots clés : Identité. Altérité. Inconscient. Freud. Lacan.

* Artículo recibido el 2/02/2019, aceptado el 2/12/2019. 


\section{Introduction}

Il faut tout mettre en mots. C'est comme ça qu'on neutralise le mal. Quand on nomme, l'événement perd de sa vitalité. Je viens d'une société marquée par l'oralité, mais certaines paroles ne s'énoncent pas; celles qui font mal, sur le plan individuel ou collectif. Verbaliser une chose risque de la créer [...]. Mais ce dont on ne veut pas parler se répète par des modalités différentes (Miano, $2016: 34-35$ ).

Ces paroles de Léonora Miano prononcées en 2016, lors de la parution de son roman Crépuscule du tourment, aux éditions Grasset, montrent à quel point l'oralité prend une place importante dans son œuvre.

Cet article sera consacré aux termes d'adresse dans L'intérieur de la nuit de Léonora Miano. Suivant une approche psychanalytique et sémantico-pragmatique, cette contribution tentera de montrer la façon dont les termes d'adresse, en tant que marqueurs discursifs d'oralité, participent à la construction de l'identité, ainsi qu'à la monstration de la "pulsion invocante " (Lacan, 1973), miroir de l'inconscient.

Pour ce faire, notre corpus, numérisé de 85 occurrences $^{2}$, comprend des termes d'adresse issus des dialogues du roman précité.

À partir des travaux de Catherine Kerbrat-Orecchioni (1992, 2010), cette recherche se situe dans la lignée des réflexions de Delphine Perret $(1968,1970)$ et Dominique Lagorgette (1998, 2003, 2004, 2007). D’autres publications de Michèle Monte $(2005,2008)$ ou Anna Jaubert (2017) ont nourri ma recherche. Concernant le domaine de la psychanalyse, les travaux de Freud $(1966,1968,1981)$ et de Lacan (1973) ont permis de relier ce domaine à celui de la pragmatique.

Les termes d'adresse ont fait l'objet de nombreuses études et publications dont Perret (1968, 1970), André-Larochebouvy (1980), Braun (1988), KerbratOrecchioni (1992, 1995, 2010), Neveu (2003, 2005), Lagorgette (1998, 2003, 2004, 2007, 2009), Coffen (2002), Lagorgette et Larrivée (2004), Détrie (2006), Béal (2009), Lehmann (2010), Denoyelle (2016), ou Jaubert (2017), pour n'en citer que quelques-unes. On ne reprendra ici ni leurs définitions ni leurs diverses taxonomies, la littérature en est abondante.

Les marques d'oralité « renvoient à un locuteur, elles donnent à voir l'attitude de celui-ci par rapport à son propre discours, ses rapports avec l'allocutaire, les stratégies argumentatives qu'il met en place» (Rodríguez Somolinos, 2016 : 2). Parmi les

\footnotetext{
${ }^{1}$ L'auteure a publié sous la dénomination de "Suite africaine" trois romans: L'intérieur de la nuit (2005), Contours du jour qui vient (2006) et Les Aubes écarlates (2009).

${ }^{2}$ Ce corpus, qui ne vise pas à l'exhaustivité, est tiré d'un autre (corpus) encore plus vaste (120 occurrences). Pour cette contribution, les occurrences seront tirées notamment du premier roman.
} 
marqueurs d'oralité, les termes d'adresse ${ }^{3}$ témoignent de cette attitude relationnelle largement étudiée par Kerbrat-Orecchioni (1992 : 15), qu'elle définit en ces termes : " un ensemble d'expressions dont dispose le locuteur pour désigner son allocutaire».

Trois dimensions relationnelles, d'après l'auteure (Kerbrat-Orecchioni, 1992 : 35), organisent l'interaction entre les interlocuteurs :

1. Axe "horizontal » ou la distance, plus ou moins grande, instaurée entre les interlocuteurs, les termes d'adresse permettant d'établir des distinctions fines entre la familiarité (qui peut aller jusqu'à l'intimité), la solidarité ou l'informalité (Orecchioni, $1992: 35)$.

2. Axe "vertical», qui concerne les relations de pouvoir : la relation d'égalité/inégalité entre les interlocuteurs, c'est-à-dire si l'emploi des termes d'adresse est symétrique, ou au contraire dissymétrique (Kerbrat-Orecchioni, 1992: 35). Cet axe, nous le verrons, est notamment saillant dans notre corpus, de par la nature de la relation des interlocuteurs.

3. Axe qui précise le caract ère "consensuel ou conflictuel» (KerbratOrecchioni, 1992 : 35) de la relation interpersonnelle.

Les termes d'adresse manifestent ainsi cette double relation des interlocuteurs qui «a pour fondement la définition de chacun comme être humain assumant telle fonction sociale » (Perret, 1968: 10). Leurs différents rôles ont déjà été étudiés : rôle désignatif (Lagorgette, 1998), rôle prédicatif (Lagorgette, 2003), rôle sociolinguistique (Perret, 1970), rôle argumentatif (Détrie, 2006 ; Monte, 2008). Leur forme morphologique « renvoie à un degré de conventionalisation, de l'inspiration conjoncturelle d'un locuteur aux emplois pérennes liés aux routines socio-discursives", d'après Jaubert (2017: 4).

Les travaux qui ont fait date sur les appellatifs depuis les années soixante ont porté sur des textes notamment littéraires. Traiter les termes d'adresse dans un texte littéraire d'un point de vue psychanalytique n'est pas chose aisée, ne serait-ce qu'en raison de l'hétérogénéité innée à ces marqueurs d'oralité qui demeurent morcelés en autant de formes, d'aspects qu'il y a d'approches. La parole (et la voix) est au coeur de la cure analytique (la psychanalyse), mais aussi de l'interpellation dont la pragmatique est chargée 4 . Il n'y a que le discours qui dote les unités de la langue d'une catégorie

\footnotetext{
${ }^{3}$ Je préfere «terme d'adresse», emprunté à Delphine Perret (1968) ou à Dominique Lagorgette (2003), à d'autres dénominations, telles que : vocatif, apostrophe, nom d'adresse ou appellatif. Pourtant les termes «appellatif » ou "vocatif " seront également employés dans cette contribution afin d'éviter des répétitions. Quoique le terme d'adresse "désignatif " ne soit pas exclu de cette recherche, les occurrences repérées dans ce travail se focalisent sur des appellatifs exclusivement "allocutifs », au sens de Perret (1970).

${ }^{4}$ Marina Aragón Cobo (1998), dans son article, très éclairant, intitulé « Les appellatifs en approche pragmatique. Didactique de leur traduction dans un corpus théâtral », montre la façon dont les appel-
} 
fonctionnelle, le terme d'adresse en faisant preuve: quoique certaines unités de la langue soient plus susceptibles que d'autres de devenir des interpellations, ce n'est qu'en discours qu'elles le deviennent, au sens de Kerbrat-Orecchioni.

Les appellatifs d'un texte littéraire ne diffèrent pas beaucoup de ceux issus des discours oraux. Michèle Monte (2008 : 1417-1418), qui a étudié l'apostrophe dans les textes littéraires, affirme que ces procédés :

[...] fonctionnent de façon très semblable à celles des discours témoins et jouent un double rôle de structuration du discours et de construction de la coénonciation, assez proche de celui qui a pu être dégagé pour les particules énonciatives ou les intonèmes par les travaux menés sur la structuration discursive à l'oral, notamment Roulet et al. (1985) et Morel et DanonBoileau (1998).

Des écrivains tels que Goethe, Shakespeare ou Schiller ont exercé une influence incontestable sur Freud. En 1935, il note dans une lettre à Thomas Mann : "Les mots de l'écrivain sont assurément des actes " (cité par Assoun, 1996 : 109). Si les représentations de mots sont les restes mnésiques de choses, autrefois des perceptions, qui ont laissé des traces d'inscription, la mise en mots de ces perceptions ou événements, à travers la mémoire et la narration, devient le porte-parole de la voix des autres, en l'occurrence de la voix de Miano, nous le verrons. "La liberté poétique, loin d'être invention arbitraire, est l'aptitude à soutenir à la fois la scène poétique et son étayage sur la réalité [...]» (Assoun, $1996: 110$ ).

Les propos ci-dessus permettraient d'appuyer que les termes d'adresse, issus de textes littéraires, fonctionnent de façon similaire aux appellatifs des interactions verbales des discours oraux. Dans la psychanalyse, par exemple, le transfert opère une mise en scène de l'énonciation entre le patient et l'analysant. Ce phénomène (le transfert) "s'établit spontanément dans toutes les relations humaines, aussi bien que dans le rapport de malade à médecin » (Freud, 1966a : 62) et "domine toutes les relations d'une personne donnée avec son entourage humain " (Freud, 1968: 53).

La nature orale et spontanée du terme d'adresse, son hétérogénéité, ainsi que son côté pulsionnel ${ }^{5}$ invitent à le situer dans une mouvance large et à s'interroger sur sa dimension psychanalytique, en particulier sur la «pulsion invocante » (Lacan, 1973: 182). On peut formuler l'hypothèse que le terme d'adresse relève de l'inconscient à travers la pulsion invocante qui se cristallise dans la parole (et la voix)

latifs relèvent nécessairement de la pragmatique énonciative, la pragmatique illocutoire et la pragmatique conversationnelle.

${ }^{5}$ La preuve en est la correction venant de la part de l'interlocuteur qui parfois refuse que l'on s'adresse à lui de telle manière ou telle autre, par telle forme appellative. Disons qu'il ne peut s'empêcher parfois, pour des raisons affectives, de corriger son interlocuteur. 
des interlocuteurs. Cette pulsion fut considérée par Lacan, (1973: 96), parmi toutes les pulsions, "la plus proche de l'expérience de l'inconscient ", la voix étant objet $a^{6}$.

L'emploi des termes d'adresse est intimement lié à la représentation que l'on a de soi-même. La double relation qu'ils déclenchent entre les interlocuteurs entraîne un positionnement social et relationnel venant de leur part. Leur usage implique non seulement la relation à la personne à qui l'on s'adresse, mais l'acte de se faire adresser à l'autre, de qui, à son tour, on obtient un retour de paroles ; ce n'est qu'en imaginant une pareille situation - où on se fait adresser - que l'on pense (la présence de) l'autre. Dans ce sens, aborder les termes d'adresse depuis la dimension de l'altérité et leur rapport à l'inconscient permet d'envisager autrement la nature de la relation des interlocuteurs, leur identité se constituant dans le rapport à l'autre auquel chacun d'eux tient par nécessité. Dans les occurrences relevées, on verra la façon dont l'identité (la négociation des identités) des interlocuteurs se construit au fur et à mesure de leur interaction relationnelle.

Ceci dit, je m'attarderai tout d'abord au concept de l'«autre», qui dans la psychanalyse est fondamental pour comprendre la façon dont le sujet parlant établit sa relation à l'autre/Autre, qui le détermine et dans sa parole et dans son évolution psychique.

1. Altérité et termes d'adresse : les trois temps grammaticaux de la pulsion invocante

C'est Lacan (1978) qui, dans Le Moi dans la théorie de Freud et dans la technique psychanalytique (Séminaire, Livre II), a distingué l'autre (le " petit autre ») de l'Autre (le " grand Autre "). Il convient de rappeler que l'altérité désigne le " caractère de ce qui est autre» (Lalande, 1996: 39). Cette notion philosophique caractérise l'individu dans une relation basée sur la différence, où celui-ci construit son identité dans son rapport à l'autre et à sa parole ; c'est avec l'autre, avec autrui que je suis conscient de mon existence, je me saisis en tant qu'être existant, et, par corollaire, je saisis l'existence de l'autre. Si la psychanalyse n'exclut pas cette définition de l'autre (celui qui n'est pas moi), elle envisage "l'autre» (le petit autre) en tant qu' " une projection de l'ego [...] dans l'ordre de l'imaginaire" (Cléro, 2003: 145-146). Lacan a ainsi distingué "l'autre» de «l'Autre» (le grand Autre), c'est-à-dire «l'altérité radicale» ou Autre qui, dans l'ordre du symbolique, constitue le discours de l'inconscient, "l'autre scène»; cet Autre par lequel les refoulements échappent au sujet parlant, mais qui rend possible la signification. Un passage de Lacan, issu du Séminaire III, permet d'éclairer que la parole c'est faire parler l'autre : "À l'intérieur de la notion de communication en tant que généralisée, je spécifie ce qu'est la parole en tant que parler à l'autre. C'est faire parler l'autre comme tel. Cet autre, nous

${ }^{6}$ L'objet a est pour Lacan (1973) « l'objet cause du désir ». Je reviens sur ce sujet ultérieurement. 
l'écrirons avec un grand A [...] quelle est cette part, dans le sujet, qui parle? L'analyse dit c'est l'inconscient. Naturellement, pour que la question ait un sens, il faut que vous ayez admis que cet inconscient est quelque chose qui parle dans le sujet, au-delà du sujet, et même quand le sujet ne le sait pas, et qui en dit plus qu'il ne le croit".

Le terme d'adresse indexe la présence de l'autre dans le discours, puisque la façon dont on construit la parole (l'intonation, les connecteurs, le balisage métalinguistique ${ }^{7}$, etc.) suppose nécessairement la présence d'un interlocuteur et la supposition d'être adressé. Delphine Perret $(1968: 3)$ rappelle que « l'existence du vocatif est donc l'un des fondements de la communication ». Mais si l'expérience d'être adressé est essentielle et nécessaire pour l'être humain, elle est à la fois problématique. Le sujet parlant se constitue dans et par le langage. La reconnaissance de la parole de l'autre constitue un départ. L'autre est mon semblable, mais il est aussi mon opposé, mon contraire au sein de l'altérité discursive : celui qui dit " je» se sait interpelé par un autre qui, différent de moi, est " tu », et réciproquement. Tel que mentionné plus haut, étant donné que la manifestation des termes d'adresse correspond à la pragmatique, la psychanalyse, dont la parole est au coeur de sa pratique clinique, aurait à plus forte raison son mot à dire. Pour Freud, l'identité se construit au sein de l'altérité "dans le conflit : entre l'identité pour soi et l'identité pour autrui, d'une part ; entre les différentes instances de l'individu que sont le Ça, le Moi et le Surmoi, d'autre part» (Oppenheimer, 2002 : 783-784). Elle résulte ainsi « de la reconnaissance réciproque du moi et de l'autre, elle naît d'un processus conflictuel où se construisent des interactions individuelles, des pratiques sociales objectives et subjectives " (Taylor, 1998 : 14-23). Les termes d'adresse cristallisent magistralement ces rapports relationnels conflictuels au sein des interactions verbales. Pour Dominique Lagorgette (2007 : 116), la notion d'identité est toujours associée aux traces qui déterminent les relations interdiscursives :

[...] qu'elle soit nationale, sociale, ou individuelle, [l'identité] est fréquemment associée [...] aux marques qui régissent les rapports interpersonnels. Le fait de choisir telle ou telle forme d'adresse sera, par exemple, le premier indice pour l'allocutaire d'un certain degré d'intégration du partenaire dans le jeu social.

Parmi d'autres, la preuve en est l'insertion des rectifications venant de la part de l'interlocuteur qui parfois refuse que l'on s'adresse à lui de telle manière ou telle autre, par telle forme appellative. Disons qu'il ne peut s'empêcher, pour des raisons affectives proprès à son discours, de corriger son interlocuteur. Comme corollaire, le

${ }^{7}$ Dans ce cas, ce serait l'oral transcrit par l'écrit. 
choix des termes d'adresse obéirait, outre à des impératifs pragmatiques, à une pulsion dite "invocante ", d'après Lacan (1973 : 182), dans la mesure où la parole n'existe que pour l'autre. Anna Jaubert (2017: 5) signale que «le trait commun des appellatifs réside non pas dans un champ conceptuel spécifique, mais dans la motivation qui inspire le choix de tel ou tel appellatif, plutôt que d'un autre ». Cette motivation tient de la relation (affective) des interlocuteurs au moment où l'énonciation a lieu. La pulsion invocante mobilise ainsi la parole (investie d'affect) que l'on adresse à l'autre, cet affect étant dérivé de la "pulsion ${ }^{8}$ (Kristeva, $2010: 2$ ).

Comme je viens de l'évoquer, c'est Lacan qui a introduit le terme de pulsion invocante, mais il n'a pas développé le concept. Par la suite quelques psychanalystes lacaniens y ont réfléchi, dont Jean Michel Vives, qui, dans «À l'entour de la pulsion invocante ", remarque :

Invocare, en latin, renvoie à l'appel. Le circuit de la pulsion invocante se déclinera donc entre un "être appelé ", "se faire appeler » $[\ldots]$, « appeler ». Mais pour appeler, il faut donner de la voix, la déposer comme on dépose le regard devant un tableau. Pour cela, il faut que le sujet l'ait reçue de l'Autre, qui aura répondu au cri qu'il aura interprété comme une demande, puis l'ait oubliée afin de pouvoir disposer de sa voix sans se trouver encombré de celle de l'Autre (Vives, 1999-2000 : 143).

C'est dans le Séminaire (livre IX) sur « L'angoisse » que Lacan parle de la voix, en particulier de la voix ${ }^{9}$ qui réclame obéissance. Il parle de la pulsion invocante dans le Séminaire, livre XI sur "Les quatre concepts fondamentaux de la psychanalyse » (Lacan, 1973 : 182), pour reprendre ce qu'il disait dans les pages précédentes sur la pulsion définissant la voix comme objet du désir. Cette pulsion serait pour Lacan (1973 : 96) "la plus proche de l'expérience de l'inconscient». Ce qui est intéressant pour mon objet, les termes d'adresse, c'est que les trois temps ${ }^{10}$ grammaticaux de la pulsion s'accomplissent : appeler ${ }^{11}$ (actif), être appelé (passif), se faire appeler (causation réflexive et passivation). Mais si l'on suit le circuit de la pulsion invocante, il faudrait tenir compte que lorsqu'on interpelle quelqu'un, l'autre doit entendre nécessairement : appeler ou entendre (actif), être appelé ou être entendu (passif), se faire appeler ou se faire entendre (causation réflexive et passivation). Ce troisième temps est ce qui fait de la pulsion invocante un appel vers l'Autre par où le sujet se fait objet

\footnotetext{
${ }^{8}$ L'affect « inclut aussi bien les sentiments de plaisir que de déplaisir « dont le Moi se débarrasse [...] par le moyen d'une activité psychique " (Green 1973 : 27, cité par Kristeva).

${ }^{9}$ Je remercie le psychanalyste Jean-Jacques Barreau de son aide sur cette question.

${ }^{10}$ En linguistique, on parle de la voix (active ou passive), alors qu'en psychanalyse il s'agit de temps grammatical de la pulsion.

${ }^{11}$ Interpeller quelqu'un.
} 
du désir de l'autre. Le terme de pulsion invocante revient, chez Lacan (1976), dans le Séminaire XXIV, L'insu que sait de l'une bévue s'aile à mourre, en particulier dans l'intervention d'Alain Didier-Weill qui fait de l'écoute plutôt que de la voix ${ }^{12}$ l'objet de la pulsion; jusqu'à parler d'une "pulsion d'écoute». Une des particularités de la pulsion d'invocation par rapport aux autres pulsions partielles, c'est que, dans le mouvement de ses trois temps grammaticaux, son organe source n'est pas le même : si voir, être vu, se faire voir concerne à chaque fois l'œil, se faire appeler ou se faire entendre ne concerne pas que (l'organe de) la bouche, mais aussi l'oreille, une bouche-oreille.

Avant de montrer les premières occurrences de mon corpus, je me permets de présenter brièvement l'auteure :

Écrivain femme d'expression française (franco-camerounaise), Léonora Miano est née en 1973, à Douala (Cameroun) où elle passe son enfance et son adolescence jusqu'à l'âge de 18 ans. En 1991, elle s'installe en France pour étudier la littérature anglo-américaine. Elle a publié plus d'une quinzaine d'ouvrages (romans, nouvelles, essais, théâtre, etc.) qui lui ont valu de nombreux prix et des distinctions littéraires. Son premier roman, L'intérieur de la nuit, fut publié en 2005, chez Plon, et a reçu six prix. Contours du jour qui vient, son deuxième roman, a obtenu, en 2006, le prix Goncourt des lycéens. C'est avec La Saison de l'ombre (2013) qu'on lui a décerné le Prix Femina.

\section{Les termes d'adresse dans L'Intérieur de la nuit}

Dans L'intérieur de la nuit l'histoire se passe dans un village imaginaire d'Afrique noire: Eku. Ayant quitté l'Afrique pour faire ses études en Europe, Ayané, la « fille de l'étrangère ${ }^{13}$, retourne dans son village pour être au chevet de sa mère mourante.

Des miliciens sanguinaires sont venus au petit village d'Eku afin de soumettre son peuple à leur volonté, dans le but de réhabiliter une Afrique mythique. Ayané sera témoin d'une scène atroce de cannibalisme imposée par les rebelles qui sacrifient un enfant du clan pour le décapiter et, par la suite, le manger.

En novembre 2005, Miano explique, dans un entretien, Les interviews d'Amina, comment et pourquoi lui est venu l'idée sur cette histoire de cannibalisme :

J'avais vu un reportage sur la cinquième, il y a deux ans, sur les enfants soldats dans l'est du Zaire. On y interrogeait un adolescent de quatorze ans et on lui demandait pourquoi il était de-

\footnotetext{
12 Pour sa part, Claire Gillie (2016), psychanalyste, musicologue, musicienne, et spécialiste de la voix, a aussi étudié la voix et son rapport à l'inconscient.

13 Cette dénomination est récurrente tout au long du roman qui apparaît aussi en tant qu'interpellation.
} 
venu enfant soldat. Il racontait qu'un jour des miliciens étaient arrivés dans son village. On avait tué son petit frère, on avait obligé la population à le manger et il avait la rage. Une question m'a obsédée : pourquoi les gens ont-ils accepté de manger cet enfant? Je voulais comprendre les mécanismes mentaux qui expliquent comment on peut commettre un acte aussi horrible. Pourquoi on l'accepte et comment on vit avec...» (Miano 2005b : 68).

D’après Miano, cette scène est « comme une métaphore des dangers du retour forcené aux valeurs ancestrales. Les villageois acceptent cette abomination parce qu'ils ont peur, parce qu'en Afrique on ne combat pas le mal : on s'y soumet avec l'espoir de lui survivre», tel qu'elle l'a déclaré, dans un entretien réalisé par Paul Yange (2006 : en ligne).

On verra que les dénominations employées tout au long du roman restent plus ou moins stables. Si d'un côté, les personnages s'interpellent au moyen de leurs prénoms ou par le biais de termes affectifs, d'un autre côté, ils le feront au moyen des formes nominales d'adresse désignant notamment leur position hiérarchique. Dans les quatre premiers exemples, on observe que le terme d'adresse apparaît de préférence en début ou en fin de dialogue. En début de dialogue, dans le but d'instituer l'allocutaire et assurer leur échange dialogal. L'auteure cherche constamment à reproduire le style oral propre au discours rapporté direct des interactions verbales. Dans le premier exemple du corpus, on peut constater que la construction du système d'adresse est conditionnée culturellement :

(1) Ayané avait trouvé Ito et Isadi, deux femmes du village, dans la case de sa mère. Elles venaient de lui faire sa toilette et de lui donner un peu de bouillon. La conversation avait été brève. La fille d'Aama avait seulement dit:

-le vous salue $e^{14}$, mes tantes.

Et elles avaient répondu :

-Bonne arrivée!

Elle s'était ensuite approchée de sa mère qui dormait à présent, épuisée par les efforts qu'elle avait dû faire pour manger. Elle lui avait embrassée le front et lui avait tenu la main. Sans regarder les femmes, elle leur avait demandé [...] (Miano, 2005 : 25).

Aama, la mère de la protagoniste (Ayané), est gravement malade. Après une longue période en Europe, sa fille retourne dans son village natal (Eku) pour être au chevet

\footnotetext{
${ }^{14}$ L'italicisation appartient à l'auteure qui cherche à bien marquer le discours du récit. C'est moi qui surligne en noir les termes d'adresse dans chaque extrait.
} 
de sa mère. C'est au moyen de ce marqueur discursif ( «mes tantes ») que la protagoniste appelle les amies de sa mère. Le choix de ce terme d'adresse est d'emblée le premier paramètre pour l'allocutaire de l'intégration de l'interlocuteur dans l'interaction sociale. On sait que les termes d'adresse, en tant que porteurs de valeurs a fordles

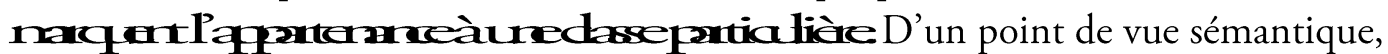
ce terme d'adresse exprime une valeur sociale et relationnelle, et montre, tel que je viens de l'évoquer, que le système d'adressage est conditionné culturellement : dans certaines sociétés africaines, c'est l'usage d'appeler les personnes proches au moyen d'un terme relationnel affectif relié à la parenté. En l'occurrence, Ayané s'adresse à des amies voisines de sa mère au moyen de ce terme relationnel affectif : le terme de parenté «tante» + le déterminant possessif (mes), alors qu'elles n'ont pas un lien de sang. Depuis l'axe horizontal, la distance instaurée entre elles est apparemment étroite, la raison n'étant que culturelle et motivée implicitement par la protagoniste qui est censée être au courant des connaissances proches de sa mère. La valeur lexicale de ce terme d'adresse ne suffit pas à identifier l'acte produit où il apparaît, au sens de Lagorgette (2003 : 50). Le contexte, qui rend transparente la distance interpersonnelle, justifie l'emploi pragmatique du déterminant possessif dont la signification ne s'actualise qu'au sein de cette société africaine aux traditions ancestrales. L'auteure qui insère, de manière récurrente, tout au long du roman, ces types d'apostrophes, chercherait ainsi à souligner la mémoire culturelle et discursive appartenant à la société africaine (camerounaise) dont elle provient :

(2) Mais il n'y avait plus rien à faire. Il ne servait à rien de s'excuser, puisqu'elle n'avait rien pour les villageois. Pour couronner le tout, lorsqu'Aama avait ouvert les yeux des heures plus tard, alors que le soir tombait, cela avait été pour dire :

-Ayané, je vais mourir (Miano, 2015 : 27).

Dans (2), c'est un nom propre (Ayané) qui témoigne dans ce contexte de l'intimité. L'apparition ici du terme d'adresse tient à la nature de l'acte illocutoire effectué : plus l'acte illocutoire est transcendant pour la relation des interlocuteurs, plus il impliquera l'allocutaire, motivant la manifestation de ces formes d'adresse. Si s'adresser à son enfant au moyen de termes d'adresse affectifs est un acte naturel et spontané, l'appeler par son nom devient, en revanche dans certains contextes la manifestation verbale d'une affliction à partager. C'est ce qui confère, en l'occurrence, d'un point de vue sémantique, un caractère solennel à l'interaction verbale, outre la conséquence qui en dérive de manière imminente : la mort de sa mère.

D'un point de vue pragmatique, cet acte de langage accomplit l'activité d'adressage depuis l'axe horizontal, permettant d'expliciter le lien de familiarité, le rapport d'intimité entre la mère mourante et sa fille. L'emploi de l'appellatif (ici un nom propre) «Ayané » fait preuve de la réciprocité de l'altérité ; il projette une image 
de l'autre, et spéculairement, une image de soi ; s'adresser à l'autre de qui on obtiendra un retour de paroles implique que l'on participe de l'identité de l'autre. « [L]'amour appelle réciprocité, et la réciprocité suppose une égalité » (Jaubert, 2017 : 10).

Lisons l'extrait suivant:

(3) Ayant déjà enfreint les règles les plus élémentaires de la bienséance, elle avait fait taire ses réticences pour se rendre chez celle qui était devenue la doyenne du village, et qui lui avait toujours été tellement hostile. Elle avait dû traverser tout le village, se présenter les mains vides dans la concession d'Ié dont les brus affairées ne lui avaient pas accordé d'attention, et discipliner la vibration de sa voix lorsqu'il avait fallu dire:

-Mère, je te salue. Je suis de retour pour quelques jours...

-Je l'ai entendu dire, en effet (Miano, 2015 : 27).

Dans (3), Ayané, la protagoniste, va chercher sa tante pour lui faire part du voeu de sa mère. Celle-ci réclame la présence de sa fille à son chevet, lors de sa mort. Ayané, s'adressant à sa tante, l'appelle « mère ». Ce terme relationnel de parenté précise au sein de l'axe 3, d'après Kerbrat-Orecchioni (1992), le caractère conflictuel de la relation interpersonnelle entre le locuteur (Ayané) et l'allocutaire, «sa tante », appelée ainsi par la protagoniste, et avec qui elle a toujours entretenu un rapport difficile. Outre cette affection de Miano pour la préservation de la mémoire culturelle et ancestrale de sa terre natale, cette situation contextuelle rappelle les tabous à l'égard du nom, et des liens de parenté des clans ou des tribus d'autrefois (en Australie, par exemple), et dont parle Freud dans Totem et tabou. Appeler sa "mère " sa tante, ce n'est pas un terme d'adresse sémantiquement " neutre ", au sens de KerbratOrecchioni $(2010: 28)$ qui a toujours insisté sur cette valeur sémantique conférée à l'interpellation.

Permettant au locuteur d'instituer son allocutaire en tant que tel, le terme d'adresse apparaît notamment en position initiale. Marina Aragón (1998 : 280) met en évidence leur "fonction d'ouvreurs de conversation", qu'elle appelle "lubrifiants ». Eu égard à la portée accordée par Léonora Miano à l'oralité, on peut constater à quel point elle veille à démarquer le discours rapporté du récit par le biais d'un balisage métalinguistique : elle italicise tout dialogue au long des romans, outre pour le distinguer du récit, pour bien souligner au lecteur l'usage d'un discours oralisé :

(4) -Ma pauvre, la vie, ce n'est pas comme ça (Miano, $2005: 42$ )

(5) Diamant était donc une bombe sexuelle, et la manière dont elle croisait ses mains aux ongles longs, apanage de la femme qui n'avait pas à s'user les phalanges au travail, attestait qu'elle n'en ignorait rien. Elle battit des paupières, ouvrit une bouche carmin, et susurra : 
-Alors, ma puce, qu'est-ce que Tata peut faire pour toi? (Miano, 2005 : 47-48).

L'investissement affectif déclenché par les termes d'adresses ci-dessus est manifeste. Kerbrat-Orecchioni (2010 : 29) a souligné l'engagement émotionnel du locuteur dans son énonciation. Dans le cas des exemples montrés, si l'on supprime, dans le premier exemple, l'appellatif " ma pauvre ", on supprime à la fois la valeur affective de l'énoncé. L'affect est pour Freud un phénomène intrinsèquement subjectif, mais aussi relationnel, me semble-t-il. Dans ce sens, l'apostrophe constitue un acte de langage déclencheur des pulsions chez le locuteur, comme en témoignent, par exemple, les insultes au sein de la violence verbale, ou les hypocoristiques au sein d'un contexte familier ou d'intimité.

On remarque dans (4) et (5) comment l'écrivaine se sert, de manière recurrente, de formes nominales d'adresse $\left(\mathrm{FNA}^{15}\right)$ formées de termes relationnels affectifs ("ma pauvre », "ma puce »). Cette réitération est une pratique dialogique habituelle dans ce type de contexte familial (parental). Dans (4), la distance instaurée entre les interlocuteurs (ici Ayané parle à sa tante) manifeste un rapport de familiarité, précédemment signalé. Cette FNA vient ajouter une charge sémantico-affective s'appropriant la personne aimée, par le biais de l'appellatif " ma pauvre »; une manière pour l'allocutaire de rendre transparent sa posture subjective.

Dans (5), au moyen d'un terme relationnel affectif ("ma puce»), la FNA montre également que la relation interpersonnelle entretenue a lieu de gré à gré, étant fréquemment utilisée à l'oral. D'un point de vue pragmatique, les deux actes de langage accomplissent l'activité d'adressage depuis l'axe horizontal, permettant ainsi d'expliciter le lien de familiarité ou de cordialité entre les interlocuteurs.

On pourrait multiplier les exemples de ce genre de termes d'adresse, pourtant d'autres occurrences comportant de diverses valeurs sémantico-pragmatiques, éloignées des précédentes, seront montrées par la suite.

Les occurrences qui suivent mettent en scène l'épisode du cannibalisme, mentionné précédemment, raconté dans L'intérieur de la nuit.

Lisons tout d'abord les préliminaires et les négociations verbales servant d'entrée à l'interaction dialogique, l'invocation étant incontournable, suite à la réquisition du milicien :

(6) Le premier, ayant rejoint l'ancien, lui dit dans la langue des Blancs qu'elle ne comprenait pas bien, mais dont Eyoum, en tant que fils de notable, avait appris quelques rudiments il $y$ avait fort longtemps :

- Vieil homme, commande à tes gens de sortir, et de venir se rassembler ici.

${ }^{15}$ C'est à Kerbrat-Orecchioni (2010) que l'on emprunte les sigles. 
Prononçant ces paroles, il fit un geste de la main et ses sbires se déployèrent à l'arrière de la place, le dos tourné vers la brousse où nul ne se rendait jamais, depuis que huit hommes du clan s'y étaient évaporés. Si d'aventure on avait voulu décamper par l'orient, en direction du fleuve qui se trouvait à quelques jours de là, on se serait fait étendre d'une seule balle (Miano, 2005 : 73-74).

Les rebelles arrivent au village. Les interlocuteurs ne se connaissent pas au départ. C'est d'abord le milicien qui s'adresse au chef du clan. La FNA employée, "vieil homme", placée en tête de phrase, montre la pertinence de l'emploi des termes d'adresse dans un acte directif, en particulier les actes réquisitionnels. Vouloir imposer sa volonté implique s'assurer la présence de l'allocutaire dans le but de prévoir son objectif pour l'accomplir.

D'un point de vue syntaxique, cette FNA est marginale, au sens de KerbratOrecchioni, c'est-à-dire qu'elle n'est pas nécessaire à la complétude grammaticale de l'énoncé ; la réquisition imposée explicitement par le milicien, à l'aide du verbe "commander", et à l'impératif, invite à prendre du recul dans la réponse de l'interlocuteur.

D'un point de vue pragmatique, ce terme affectif à valeur négative ${ }^{16} s^{\prime}$ impose en tant qu'acte réquisitionnel : knilicien ici l'Aned'linarationvalsk a pis l'initiatived'établir le contact à son allocutaire, en raison de sa position dominante au sein de l'axe vertical. Placé en tête de phrase, ce terme d'adresse sert avant tout à attirer l'attention de l'allocutaire, le chef du clan (Eyoum), désigne explicitement comme le seul destinataire de la réquisition. Pourtant, son emploi, qui a lieu en public, sert à bien marquer l'autorité du locuteur face à tous ses interlocuteurs, aux gens du clan. À travers cette requête, il s'assure que son allocutaire devient son otage. La négociation des identités est d'emblée manifeste à travers la FNA "vieil homme ", sa position dominante déterminant la relation dissymétrique basée sur ce rapport de pouvoir. Cette position autoritaire est renforcée par le recours à l'impératif, ainsi qu'au ton et aux gestes mis en scène dans l'énonciation : "prononçant ces paroles, il fit un geste de la main et ses sbires se déployèrent à l'arrière de la place, le dos tourné vers la brousse ». On sait que l'axe vertical détermine les relations de pouvoir, les rela-

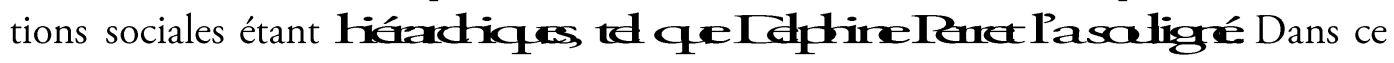

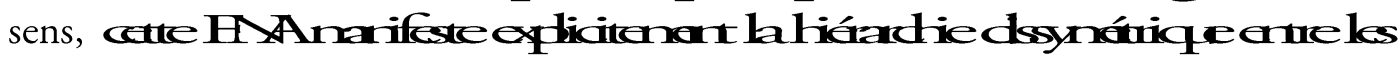

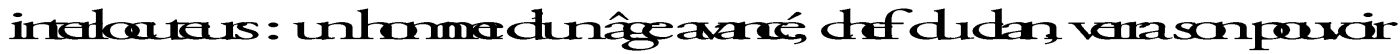

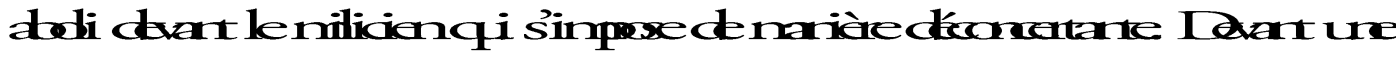

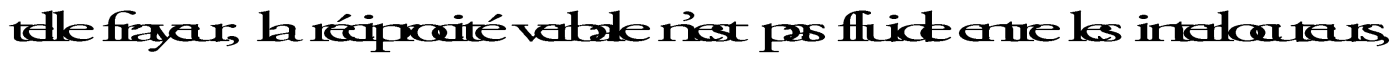

\footnotetext{
${ }^{16} \mathrm{Si}$, pour des raisons culturelles, on peut lire « vieil homme » sans le prendre au pied de la lettre, et en lui conférant une valeur désignative, sa valeur dépréciative est évidente.
} 


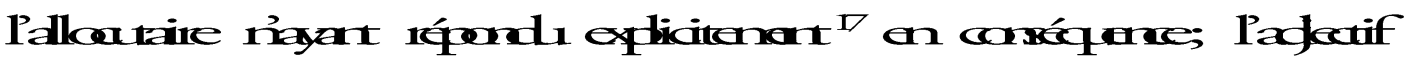

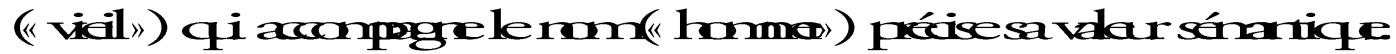

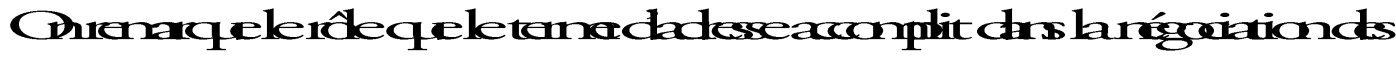
idnnités dúannimainsilabiéachie

Si l'expérience de s'adresser à l'autre ainsi que d'être adressé est essentielle et nécessaire pour l'être humain, elle est à la fois problématique. Toute altérité opère, on le sait, sur un espace de tension et de résistance. Iassuncantexteciks retaios de percir sat flagantes ${ }^{18}$, la dssynánie ds rtaias híractiques

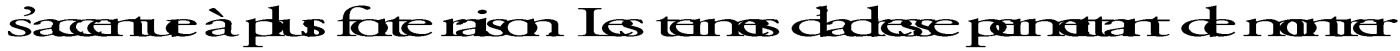

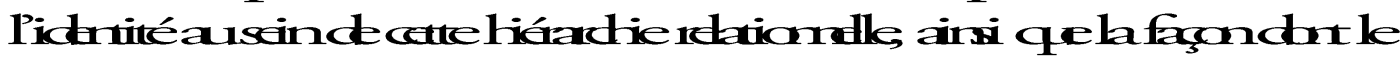

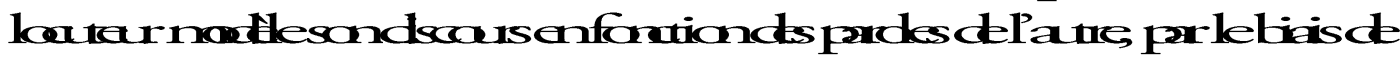

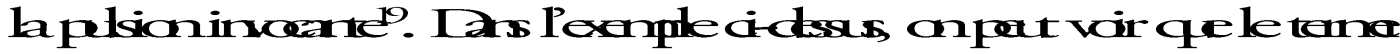

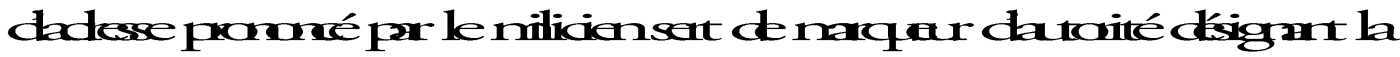
prisedepucirsurl'ane docurevidere $S$ 'adresser à l'autre implique, non seulement le désigner, mais participer de son identité qui se construit grâce à la parole de l'autre. Voici pourquoi il n'y pas de parole sans voix ; si lavixexiste ćestper Pane, aquel àlaneakpekioninceante) (Iacan 1973: 96).

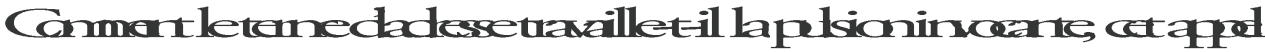
àl'ane? Iavix anksait, estundjetpe lsiand, dapès Iacan quil range

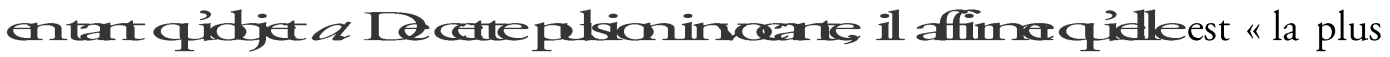
proche de l'expérience de l'inconscient" (Lacan, 1973: 96), tel que je viens de l'évoquer précédemment. Cardansetranechrs ds situ mias linites devant

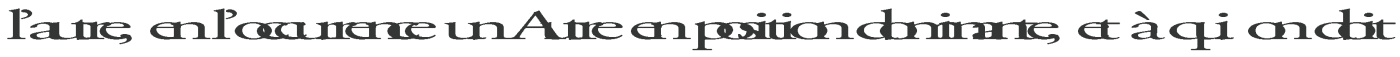

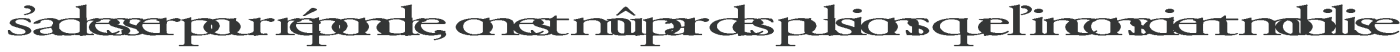

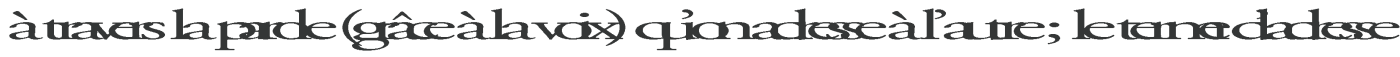

\footnotetext{
${ }^{17}$ Comme Lacan (1966 : 247) l'a signalé, le silence de l'interlocuteur ne signifie pas son absence, ce silence même est à interpréter : "II n'est pas de parole sans réponse même si elle ne rencontre que le silence, pourvu qu'elle ait un auditeur", ou encore: "La seule présence du psychanalyste apporte, avant toute intervention, la dimension du dialogue " (Lacan, $1966: 216$ ).

${ }^{18}$ Léonora Miano n’hésite pas à raconter explicitement tout au long de ses romans la barbarie d'un passé esclavagiste, ainsi que les flétrissures produites par la colonisation des Blancs en Afrique. Dans ce roman, elle répète à plusieurs reprises : "La saleté ne tue pas le Noir» (2005: 46). Dans un de ses entretiens, elle déclare: «En Afrique, quand vous êtes entre Noirs, vous n’êtes pas noire ; vous êtes vous. Et obligée alors d'entrer dans la dimension profondément humaine, et parfois spirituelle, de l'histoire du vécu des peuples (Miano, 2016 : 34-35). Pourtant, l'auteure ne soulignera pas seulement au long de ses textes les mots/maux laissés par la colonisation des Blancs, mais aussi la façon dont les Africains mêmes se sont pliés au joug de ses propres compatriotes, ayant opté pour l'obéissance, sans refuser d'obéir aux ordres imposés.

${ }^{19}$ Cette pulsion d'invocation étant transférée par la voix de l'auteure qui fait parler, à son tour, ses personnages.
} 


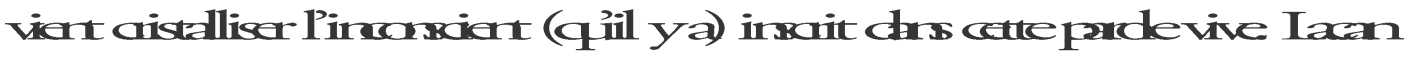

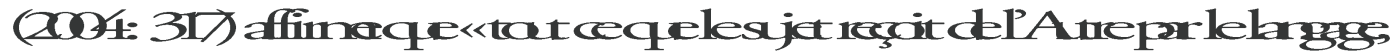

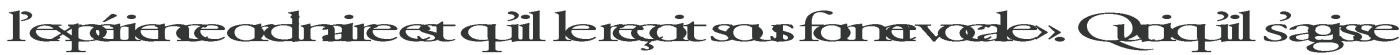

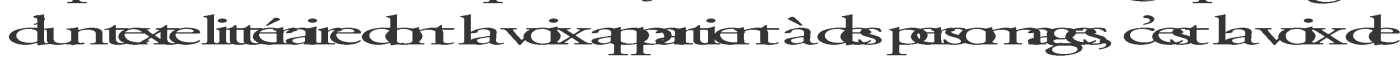

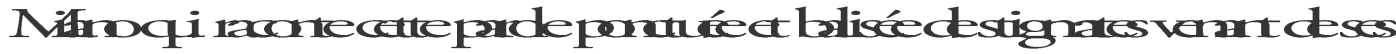
réass en Afriq $e^{20}$. Iaffarisands temas dadessechrs sanénitu redevient des restes mnésiques de son inconscient; ces restes mnésiques furent autrefois des événements réels, car la voix, en tant qu'objet pulsionnel, et agent de la parole, perdure dans la mémoire. Comprendre mon énoncé précédent, c’est comprendre la raison pour laquelle l'expression "entendre des voix " a été lexicalisée en pathologie, employée lorsque certains malades mentaux souffrent des hallucinations auditives. Pour dire à quel point la voix de l'Autre s'empare de l'inconscient et s'imprime dans la mémoire. Chez Miano la parole se trouve au coeur de l'articulation de soi et de l'autre (son peuple), qui effrayé pendant très longtemps demeure muet encore aujourd'hui ; ce peuple africain subsaharien dont la culture ne se transmet que par l'oralité et la voix des femmes. Par corollaire, l'auteure leur donne la voix aujourd'hui dans ses écrits.

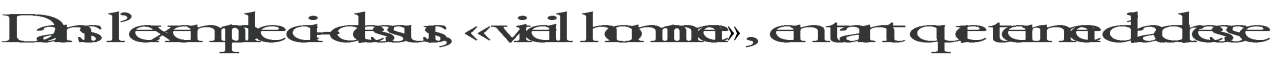

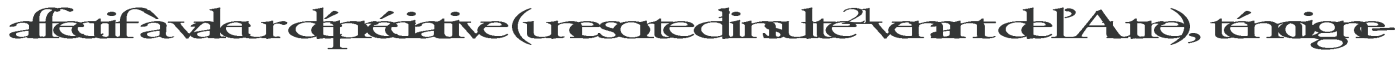

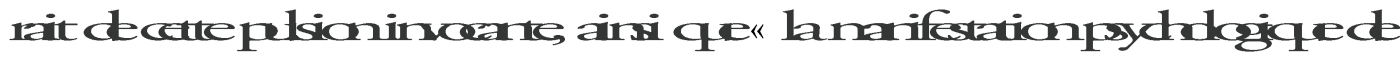

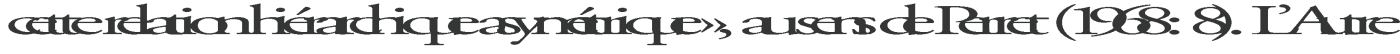

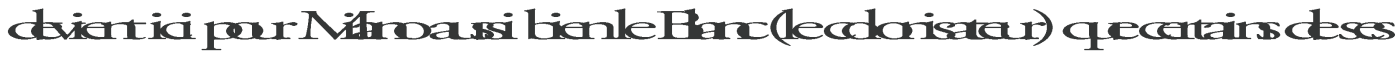

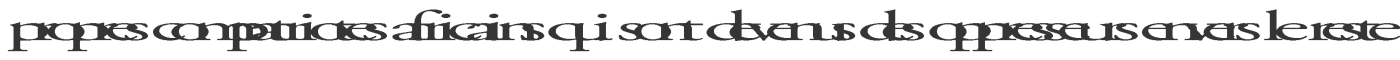

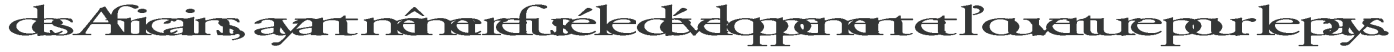
Runtant dleširsuge a ssi caneds Africairs nânes $q i$, attins defi-

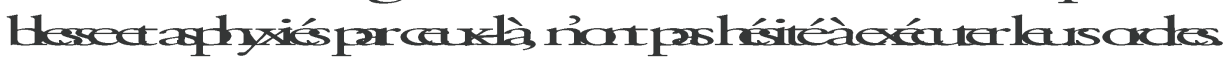

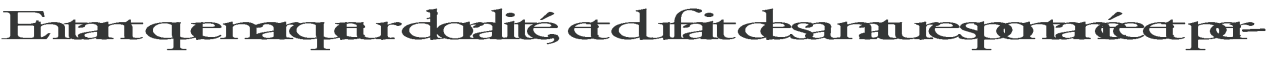
kutiveàlafín letemadadesse véhia lechrs cesers cetyedediscours, miroir de l'inconscient. Sadesseràl'autre aunojendeces fónas dsa isives

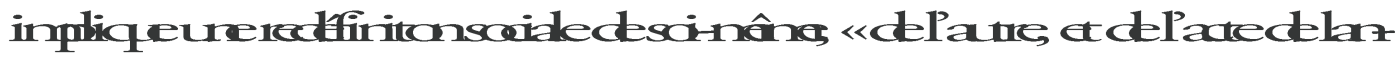
ggeqejaccomplisauseind igra per) (Iagugate, 2007: 19).

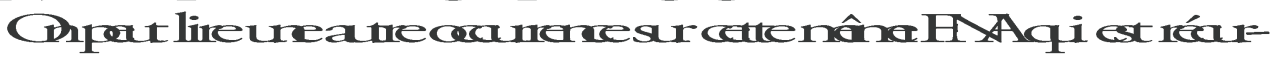
renteaulagdeceprsage:

(7) -Nous venons de l'orient, de l'autre rive du fleuve, d'un pays qu'on appelle Yénèpasi, vieil homme. Comme tu l'as entendu dire, nous avons enjambé l'eau afin de rétablir la vérité qu'ont maquil-

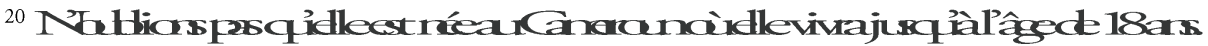

${ }^{21}$ Dominique Lagorgette et Pierre Larrivée (2004), Catherine Détrie (2006), et plus récemment Béatrice Fracchiolla (2008) ont exploré le terme d'adresse au sein de la violence verbale.
} 
lée les Blancs lorsqu'ils sont venus ici prendre possession de nos vies. Notre peuple et le tien, vieil homme, tu dois le savoir, ont un seul et même ancêtre. Notre ä̈eul commun s'appelait Ewo. Contes-tu encore aux tiens son épopée?

L'étranger aimait parler. Il se repaissait de son propre verbe, et on avait le sentiment que pour lui cette épopée était une intarissable source de ravissement. Le ton était grandiloquent, et la voix portait loin (Miano, 2005 : 77).

Ici le terme d'adresse n'est plus en tête, mais à la fin de la première proposition. Le contact est déjà bien établi de la part de l'Autre; le milicien raconte à ses interlocuteurs une histoire commune, qui leur appartient, dans le but d'être de connivence avec eux. Si dans cet exemple, le terme d'adresse n'a pas la valeur de requête comme dans l'exemple précédent, on peut constater qu'il entretient également une relation sémantiquement étroite avec les énoncés assertifs qui contiennent un acte argumentatif.

En désignant explicitement le chef du clan, le rebelle, par le biais de ce terme d'adresse, s'assure du lien frontal entre lui et l'allocutaire, gagnant par conséquent la complicité des autres ; la proposition subordonnée adverbiale de but l'entérine : "afin de rétablir la vérité qu'ont maquillée les Blancs lorsqu'ils sont venus ici prendre possession de nos vies".

On remarque que pour pour être de connivence avec ses allocutaires, il se sert du substantif Blanc, dont la signification affective est partagée entre les interlocuteurs; si la charge sémantique du mot est considérable, la connotation politique n'en est pas moins : le sujet de la race (Noir/Blanc; colonisé/colonisateur) est implicite. Il prend ainsi en compte son allocutaire l'apostrophant en souhaitant en même temps qu'il le croie. Mais il cherche à neutraliser la tentation éventuelle qu'aurait l'allocutaire (Eyoum) de faire abstraction quant à sa requête initiale. L'emploi de l'adjectif possessif dans «nos vies» est une stratégie de réparation pour tenter de désamorcer la montée en tension. A travers cette histoire ancestralement partagée, la valeur pragmatique du terme d'adresse se trouve préservée, mais sensiblement atténuée. Je réfère ici à la valeur pragmatique, appelée par Kerbrat-Orecchioni (2010:28) " interpellative diluée ", réduite à une fonction générique de renforcement. Le déterminant et le pronom possessif qui accompagnent la FNA (« notre peuple et le tien ») laissent transparaître leurs valeurs sémantico-affectives cherchant sans conteste à renforcer également le lien entre les interlocuteurs, mais aussi à atténuer un discours hypocrite.

On voit que ces formes d'adresse ont un rôle notoire dans la négociation des identités, ainsi que dans la relation interpersonnelle des interlocuteurs, dans la mesure où ils sont susceptibles de modifier leur conduite, au fur et à mesure du déroulement de l'interaction, et selon aussi leur réaction à l'écoute des paroles de l'autre. Toute 
forme d'adresse injurieuse peut se cacher sous l'hypocrisie des bonnes manières et se donner les airs d'affectation ou raffinement. Perret (1968) n'a pas negligé cette nuance à l'égard de cette affinitié entre les termes d'adresse et l'injure.

On constate qu'aborder les termes d'adresse depuis la dimension de l'altérité, sans négliger le circuit de la pulsion invocante, implique que la construction du système d'adresse est conditionnée par la parole de l'autre qui modèle constamment l'identité discursive du locuteur ; l'altérité identitaire cristallisant sans conteste. C'est dans ce sens que les termes d'adresse participent à la construction de l'identité de soi et de l'autre à travers la pulsion invocante de la parole.

Dans (8), on peut voir que le discours contient des stratégies d'éloignement par rapport à son interlocuteur. Si dans les occurrences précédentes le milicien emploie l'adjectif possessif " notre » pour s'adresser à son interlocutaire et être de connivence avec, dans l'exemple qui suit, il fera avancer son discours en ajoutant d'autres stratégies verbales pour maintenir la distance:

(8) - Nous avons oublié notre nom. Combien de temps cela fait-il, que nous n'avons plus célébré d'union entre descendants d'Iwié et d'Eku, par exemple? Voilà en un mot le pacte que nous sommes venus rétablir. Cela ne se fera pas sans mal. Si nous voulons réunir notre famille, créer enfin une entité qui portera peut-être le nom de Wonja ou de Mwayé... enfin, je n'ai pas encore vraiment décidé du nom... nous devrons nous battre, et, vieil homme, tu devras me donner des soldats! (Miano, 200 : 78).

Outre le tutoiement, déjà présent dans l'exemple précédent, le milicien s'adresse à son interlocuteur toujours au moyen du pronom "nous ", tout en insérant également le pronom «je». Il utilise aussi le pronom neutre "cela", qui témoigne d'un éloignement par rapport à son allocutaire. Ce pronom est une trace discursive de son inconscient. Ce pronom neutre est le sujet d'une proposition indépendante formulée sous la forme de la négation qui dans la psychanalyse est une affirmation, tel que l'on sait. Effectivement, ce «mal» que le locuteur annonce aura lieu par la suite. Ceci ajoute une montée en tension.

L'apparition de nouveau de cette FNA tient à sa force illocutoire conférée à l'énoncé qui implique directement son allocutaire. Cette autorité que l'Autre (le milicien) exerce sur l'autre, l'inférieur, et «cette prise de liberté dans la manière de s'adresser à l'autre signifie qu'on le considère comme un intime ", au sens de Perret (1968 : 11), ici « intime» puisque susceptible d'être maniable. Ietemacladesse

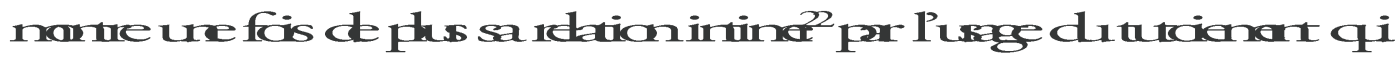
Paccanprge Cette intimité évoquée par Perret (1968: 11) «permet denommer l'autre de façon strictement personnelle. Mais elle permet aussi de l'appeler de façon

${ }^{22}$ Ici la valeur que Perret confère à « intime » s'éloigne de sa signification vulgarisée. 
multiple, selon la relation affective momentanée que le locuteur veut exprimer ». C'est le cas de ce terme d'adresse affectif à valeur négative. La FNA employée dans ce dernier exemple anticipe un moment de tension interlocutive à venir de façon plus extrème ; le rebelle tente d'influencer sur son interlocuteur (le chef du clan) se rapprochant de lui à travers une histoire commune partagée.

On peut voir comment se construit la négociation des identités entre les interlocuteurs, la position dominante (du milicien) et du dominé (du vieil homme) étant nettement marquée par l'interpellation : l'affaiblissement atteint Eyoum devant les propos du milicien.

C'est en ce sens que les termes d'adresse opèrent, Chanièredraniqu $\mathbf{~ b a}$

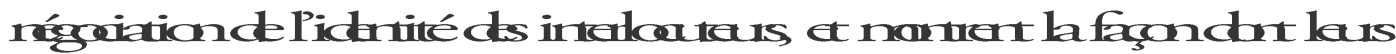

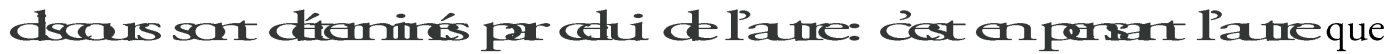
l'identité de soi se construit ; perser (aprécin) lapardedllane ćestputiaperde son identité en s'en rapprochant ou en s'en éloginant, en refusant ou en acceptant ses différences. Mais il souvent que la prévision de la parole de l'autre échoue, ce qui déclenche la béance de l'inconscient à travers précisément de la voix; Pempici $\mathbf{d e}$

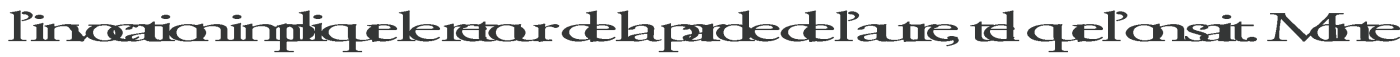
(2008 : 1421) a a ssi nis enexag el latúité chrs les tennes dadesse cane

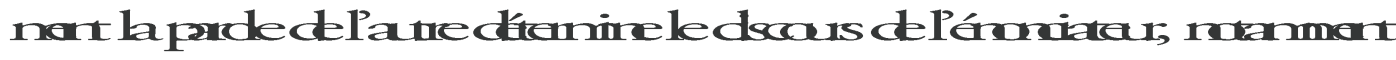
dhods ditumias decaflit: «en marquant fortement la sphère du tu, le locuteur inscrit son dire dans un dialogue et par ricochet s'énonce autre, distinct de son allocutaire». Cet «autre» dont elle parle, c'est l'autre de la pulsion invocante ; et je me permets de reprende une fois encore l'affirmation qui suit : c'est en imaginant un cadre où on se fait adresser que l'on pense (la présence de) l'autre, car le terme d'adresse joue un rôle très net tant dans l'affirmation du moi que dans le processus d'authentification. Suivant Détrie (2006), Monte considère le terme d'adresse un marqueur du passage à l'espace de la subjectivité en soi-même dans le processus d'actualisation discursive. Elle signale :

Le stade 3 [de l'actualisation discursive] correspond à l'ego (...) En un sens, il est le comble de la subjectivité. En un autre sens, il résulte de l'objectivation de soi. Le sujet se conçoit comme autre de l'autre, et doit admettre que chaque être humain, comme lui, a droit aux privilèges de la subjectivité. Cette relativité de je et l'objectivation de soi qu'elle implique conduisent à une organisation stricte de la sphère interpersonnelle et déictique, selon le mode de l'opposition et de la rupture entre les instances. La personne s'oppose à la non-personne, et à l'intérieur de la personne s'opposent je et tu (Monte, 2008 : 1422).

Dans l'exemple qui suit, le terme d'adresse traduit un acte illocutoire directif 
dans des moments stratégiques de l'interaction. Dans ce cas, la requête est formulée sous la forme d'une interrogation:

(9) -Vieil homme, où sont donc les mâles de ton peuple?

(Miano, 2005: 82).

Le rebelle demande au chef du village de choisir des mâles pour le sacrifice. Pour ce faire, il se sert de nouveau de la même forme nominale d'adresse «vieil homme ", placée cette fois en tête de phrase, le contrôle sur l'autre étant assuré. Le chef $\mathrm{du}$ clan répond à sa réquisition qu'il ne peut désobéir. La montée en tension suscitée est évidente, celui-ci refusant de livrer des batailles :

(10) -Tout de même, dix-huit de mes garçons...

-Que proposes-tu?

-Je ne sais pas. Nous n'avions pas envisagé de devoir livrer de telles batailles, et nous ne sommes pas des soldats... Rien que des cultivateurs.

-De piètres cultivateurs, si jén juge par les plants chétifs qui meurent au soleil, sur les collines (Miano, 2005 : 83-84).

Dans l'extrait qui suit la tension est manifeste, le terme d'adresse marquant nettement du mépris envers Eyoum :

(11) -Vieil homme! Tu diriges ton clan de manière déplorable! Tu es vieux, et à ce titre je te dois le respect, mais devant un tel manque de sagesse, je crois pouvoir décider sans que tu aies ton mot à dire. Tes six fils âgés de quatorze à dix-huit ans viendront donc avec moi, ainsi que trois plus jeunes ayant atteint les douze ans. J'emmène aussi avec moi neuf filles sans époux (Miano, 2005 : 84-85).

Par la suite, le chef du clan, en étant en désaccord, cherche à obtenir un revirement de la part de l'Autre :

(12) -Mais au nom des ancêtres, pourquoi te faut-il également des filles? Elles ne vont pas se battre à vos côtés, n'est-ce pas?...

-Non, en effet, elles ne feront pas partie des forces armées, mais elles peuvent nous assister de bien des façons. N'importe comment, il me faut deux ennéades des tiens, et c'est tout. Allez, debout (Miano, 2005: 85-86).

Tel que l'on peut lire, Eyoum, depuis sa position inférieure se permet de nouveau de recourir au mécanisme du tutoiement pour répondre au milicien. D'un point de vue sémantique, ce terme d'adresse («au nom des ancêtres ») renvoie à la disconvenance qui manifeste cette tension interlocutive, mais en plus, il présage un malheureux avenir. Le recours à cette apostrophe aboutit en revanche dans ce contexte à un éloignement. 
Voici une autre occurrence où l'appellatif est placé au début de la proposition :

(13) -Pourquoi voulez-vous un enfant?

[...] Le jeune homme avait l'impression que le silence épais qui s'était posé sur la place s'enroulait autor de lui, comme un boa sur le point d'etouffer sa proie. Il avait toujours voulu être un modèle pour Eyia. Il demanda une troisième fois, tentant dans un réflexe désespéré de soustraire son petit frère à l'emprise d'Ibanga. Isilo partit d'un grand rire, comme s'il avait entendu une plaisanterie aussi savoureuse qu'inopinée.

-Garçon, ce n'est pas vraiment ce qu'on appelle une attitude de guerrier. Ce serait plutôt celle d'une demoiselle craintive. Dois-je comprendre que cet enfant t'importe?

$[\ldots]$

-Ce petit garçon est mon frère. [...]

-Dans ce cas, réjouis-toi. C'est pour toi et pour tes parents que ce

sacrifice aura le plus de portée et les meilleurs effets (Miano, 2015

Dans l'exemple ci-dessus, le terme d'adresse permet de qualifier l'allocutaire de façon négative et montre à quel point le locuteur ressent le besoin de s'appuyer une fois encore sur ce genre de marqueurs qui se manifestent dans des situations bien diverses; ici au sein de l'axe 3, qui, d'après Kerbrat-Orecchioni (1992), précise le caractère conflictuel de la relation interpersonnelle. Monte (2008 : 1421) parle d'un «mouvement vers l'autre». Ce mouvement ne peut se faire que depuis la pulsion invocante où la parole manifeste le «désir de convaincre, d'arracher le consensus, plutôt qu'un repliement dans la sphère du je » (Monte, 2008 : 1421).

«Garçon", qui est employé à plusieurs reprises, acquiert ici le rang, d'un point de vue sémantique, d'insulte et le sens dépréciatif de «demoiselle», l'allocutaire ayant manifesté en ce sens explicitement son éloignement. Si dans un premier moment, on lui aurait attribué une valeur affective (positive, à nature hypocoristique), son emploi récurrent dans un tel contexte, et le ton employé que la phrase recèle, contribue à le transformer en un ordre.

Ce mouvement vers l'autre, évoqué précédemment, émane de la pulsion d'invocation où l'inconscient cristallise les termes d'adresse. Ce "quelque chose", mentionné précédemment par Lacan, parle dans et depuis la voix de l'auteure, d'où elle fait parler l'autre, mais aussi l'Autre. Si l'écriture est sans conteste un acte conscient, l'emploi de certains procédés discursifs obéirait en revanche à un acte iconscient; cette pulsion invocante, issue de l'affect, vient de l'autre et de l'Autre, cet Autre dont parle Lacan. La manifestation récurrente des appellatifs dans les dialogues des romans de Miano n'est donc pas la manifestation d'un mouvement psychique en vain, car l'auteure, on le sait, attache une grande importance à la parole, cette parole 
qui «fait taire la voix» (Vives, 1999-2000 : 146). La voix de son inconscient viendrait de l'Autre, et par corollaire de l'inconscient collectif du peuple africain, ce peuple qui est resté muet pendant si longtemps. Miano parle depuis la voix de l'autre, ses semblables, ses compatriotes, de ceux qui ont souffert des aliénations, des discriminations venant des Blancs. Mais aussi des Africains qui ont accepté le joug imposé par l'Autre (des Africains aussi), sans se révolter contre leurs propres compatriotes. Cette soumission et conformisme se cristallisent dans la subjectivation des termes d'adresse ou dans l'affect qu'ils déclenchent. Ce faisant la pulsion invocante rend transparent l'Autre dans cette relation hiérarchique dialogale. Il faut noter que l'Autre n'est pas seulement l'altérité radicale et l'inconcient qui clarifient la signification dans la chaîne des signifiants, puisque cet Autre, il faut le dire, trouve souvent son origine dans un sujet, un individu (un père dictatorial, par exemple, ou un maître de l'école qui cherche à imposer sa parole). D'autres fois l'Autre trouve sa représentation à travers (le mot) Dieu ou la religion. Miano, en tant qu'écrivaine, exerce le rôle de passeur, de médiatrice de ses vécus en Afrique. Ce n'est qu'à travers la fiction que l'auteure peut s'adresser à cet Autre qui a tellement tourmenté son peuple et la tourmente encore aujourd'hui.

Dans l'exemple qui suit, l'interaction entre les interlocuteurs s'organise toujours au sein de l'axe conflictuel. Ietennadadessepencickéthirurertation

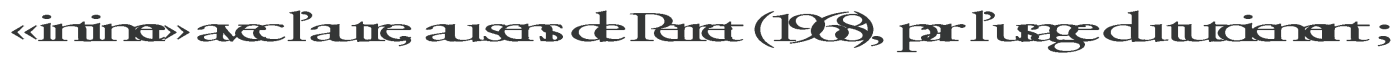
a ssi biank nilicienqelegrçanfirissent pre se toga: L'allocutaire vient d'apprendre qu'il devra manger son propre frère. À l'écoute d'une telle nouvelle, il se permet de s'adresser à l'Autre au moyen de l'apostrophe «type », terme d'adresse qui montrerait et sa force pragmatique et sa force psychologique :

(14) -Je n'ai plus de parents. Et puis, de quoi parles-tu?

-Nous allons mettre à mort ton frère, et vous le donner à manger.

$-Q u o i ?$

-Je ne suis pas surpris de ta réaction. Apparemment, ton initiation n'a pas été totale et tu ignores le sens de cet acte.

-Et je ne veux rien en savoir! J'ignore d'où tu sors, type, mais sache qu'initiation terminée ou pas, je peux t'affirmer qu'ici, il nous est interdit de manger de l'humain.

-Depuis quand?

-Depuis toujours. En dehors de certaines sociétés secrètes dissoutes depuis longtemps et dont nous nous passons!

-C'est toi qui le dis! [...]

-Pas de cette manière!

- Je ne te demande pas, garçon, je te dis. Non seulement nous le mettrons à mort, mais tu le mangeras. Et quoi que tu fasses, nous ne t'accorderons pas la satisfaction de te tuer [...] (Miano, 2005: 109). 
Dans l'occurrence ci-dessus, "garçon » est répété par le locuteur marquant de nouveau son autorité et sa position dominante. L'effet du tutoiement est particulièrement perceptible dans l'interaction verbale, non pas du fait du tutoiement du locuteur, car l'interlocuteur est un garçon effectivement jeune, mais du manque de respect manifeste dans ses paroles ; cela implique une prise de pouvoir sur l'autre, le garçon se trouvant obligé de céder à l'autorité qu'il a devant lui.

L'extrait suivant montre que l'emploi d'un terme d'adresse, inscrit dans la politesse conventionnelle, tel que "madame", est susceptible de modifier le déroulement de l'interaction dialogale. Devenant objet du discours, il provoque, dans cette occurrence, une réaction inattendue dans le comportement de l'allocutaire :

Les choses me sont apparues sous un autre angle. Je suis allée voir Kwédi, et je lui ai parlé: Madame, lui ai-je dit, ponvezvous me dire comment je pourrais me laver, et s'il serait possible d'obtenir une vraie robe et aussi une culotte? Je vous aiderai désormais comme cela m'est demandé.

Elle m'a regardée de longuement, de bas en haut et de haut en bas $[\ldots]$

Elle a répondu, en baissant les yeux sur le manioc qu'elle coupait pour le mettre à macérer dans de l'eau, jusqu'à ce qu'il fermente et qu'elle puisse le façonner en bâtonnets: $N e$ m'appelle pas madame, on n'est pas chez les Blancs, ici. Tu pourrais être ma fille, alors dit Maman Kwédi. Je lui ai dit que cela m'était impossible. Sans vouloir lui manquer de respect, j'avais déjà une mère. Elle m'a de nouveau regardée pour me demander : En es-tu bien sûre? Je n'ai rien dit. On avait dû lui raconter mon histoire. Soit, a-t-elle conclu, appelle-moi Tante Kwédi. Il y a de l'eau là derrière, dans le fût. Débrouille-toi pour m'en laisser la moitié. Nous verrons plus tard comment te vêtir (Miano, $2006: 61)$.

Ce dernier exemple montre une fois de plus à quel point un terme d'adresse, tel que "madame", est investi d'affect, la charge sémantique, ainsi que politique, étant flagrante. Cette apostrophe, qui permet à Miano de faire ressortir le sujet de la colonisation des Blancs, devient, tel que je viens de l'évoquer, " objet" du discours, donc un mot "autonyme ", au sens de Rey-Debove (1997). Parler d'un mot ce n'est pas un acte anodin. C'est donc précisément un terme d'adresse qui permet de soulever le sujet de la colonisation, l'auteure convoquant par la suite une fois encore les maux et les stigmates laissés par les Blancs en Afrique.

On peut constater comment ce marqueur d'oralité travaille la pulsion d'invocation au travers de l'affect qui émane de la voix de Miano, transférée dans ses personnages. Son inconscient mobilise, au travers de la pulsion invocante, la parole 
de l'autre/Autre, des procédés discursifs se cristallisant qui rendent transparent l'investissement affectif de la langue.

\section{En guise de conclusion}

Dans cet article, j'ai essayé de montrer la façon dont les termes d'adresse participent à la construction de l'identité des interlocuteurs, ainsi qu'à la monstration de la pulsion d'invocation. Leur l'emploi indexe nécessairement la présence d'un interlocuteur et la supposition d'être adressé. Ils sont des marqueurs manifestant passage à l'espace de la subjectivité en soi-même dans le processus d'actualisation discursive à travers la voix. C'est ici que la pulsion d'invocation intervient dans un mouvement vers l'autre depuis l'affect.

On a pu constater que les trois temps grammaticaux de la pulsion s'accomplissent dans les termes d'adresse : appeler/interpeller (actif), être appelé (passif), se faire appeler (causation réflexive et passivation). Si l'on suit le circuit de la pulsion invocante, il opère de manière réciproque un double enclenchement : appeler ou entendre (actif), être appelé ou être entendu (passif), se faire appeler ou se faire entendre (causation réflexive et passivation). Ce troisième temps/voix fait de la pulsion invocante un appel essentiel vers l'Autre/l'autre. Le terme d'adresse devient ainsi un marqueur discursif qui mobilise l'inconscient du locuteur à travers sa voix et sa parole, rebondissant nécessairement sur l'interlocuteur. S'adresser à l'autre en se faisant adresser, à travers ce phénomène discursif, implique une redéfinition de soi, mais aussi de l'autre.

\section{RÉFÉRENCES BIBLIOGRAPHIQUES}

ARAGÓN COBO, Marina (1998) : «Les appellatifs en approche pragmatique. Didactique de leur traduction dans un corpus théátral », in Teresa García-Sabell, Dolores Olivares Vaquero, Annick Boilève-Guerlet, Manuel García (éds.), Les chemins du texte : VI Coloquio da APFUE, Santiago de Compostela, Servicio de Publicaciones de la Universidade de Santiago de Compostela, 270-281.

Assoun, Paul-Laurent (1996) : Littérature et psychanalyse. Paris, Ellipses.

BÉAL, Christine (2009) : "L'évolution des termes d'adresse en français contemporain : un essai de modélisation ", in Bert Peeters et Nathalie Ramière (éds), Tu et Vous: l'embarras $d u$ choix, Limoges, Lambert-Lucas, 115-145. Disponible sur: https://hal.archives-ouvertes.fr/hal-00298549.

BRAUn, Friederike (1988): Termes of Address. Problems of patterns and usage in various languages and cultures. Berlin, Mouton de Gruyter.

CLÉro, Jean-Pierre (2003) : "Concepts lacaniens », Cités, 16/4, 145-158. Disponible sur : https://www.cairn.info/revue-cites-2003-4-page-145.htm. 
DenOyELLE, Corinne (2016) : «L'emploi des termes d'adresse dans l'oeuvre de Jean Bodel », Linx, 73 (A. Rodríguez Somolinos, dir., Énonciation et marques d'oralité dans l'évolution du français), 2-28. Disponible sur: http://journals.openedition.org/linx/1656.

DÉTRIE, Catherine (2006) : De la non-personne à la personne: l'apostrophe nominale. Paris, CNRS Éditions.

FRACCHIOLLA, Béatrice (2008) : "L'attaque courtoise : de l'usage de la politesse comme stratégie d'agression dans le débat Sarkozy-Royal du 2 mai 2007 ", in S. Heiden et B. Pincemin. Actes JADT'2008 - 9èmes journées internationales d'analyse statistique des données textuelles, Presses Universitaires de Lyon, 595-606. Disponible sur: https://halshs.archives-ouvertes.fr/halshs-00553726.

FREUD, Sigmund (1966) : Cinq leçons sur la psychanalyse. Paris, Payot.

FREUD, Sigmund (1968) : Ma vie et la psychanalyse. Paris, Gallimard.

FREUD, Sigmund (1981) : «Le moi et le ça », Introduction à la psychanalyse. Paris, Payot (Coll. Petite bibliothèque), 230-252.

GILlIE, Claire (2016) : "La voix "gorge de pigeon" », in Carine Klein-Dallant (dir.), De la voix parlée au chant. Isbergues, Éditions Klein-Dallant.

Kerbrat-OreCCHIONI, Catherine (1992) : Les interactions verbales. Paris, Armand Colin, t. II.

Kerbrat-OreCCHIONI, Catherine (1995): "La construction de la relation interpersonnelle : quelques remarques sur cette dimensión du dialogue ». Cahiers de linguistique française, 16, 69-88.

KERBRAT-ORECCHIONI, Catherine (2010) : «Introduction", S'adresser à autrui. Les formes nominales d'adresse en français, Chambéry, Université de Savoie, 7-30.

JauberT, Anna (2017): "Nommer et faire être. Pragmatique du nom propre et de l'appellatif", in Les seuils du nom propre. Disponible sur: https://hal.archivesouvertes.fr/hal-02078837.

KRISTEVA, Julia (2010) : "De l'affect ou "L'intense profondeur des mots" ». Conférence prononcée à la Facultà Teologica dell'Italia Settentrionale, Milan, 23-24 février. Disponible sur : http://www.kristeva.fr/de-l-affect.html.

LACAN, Jacques (1966) : Écrits. Paris, Seuil.

LACAN, Jacques (1973) : Le Séminaire, Livre XI (1963-1964), Les quatre concepts fondamentaux de la psychanalyse. Paris, Seuil.

LACAN, Jacques (1976) : Le Séminaire, Livre XXIV, L'insu que sait de l'une bévue s'aile à mourre (1976-1977), édition privée de l'ALI.

LACAN, Jacques (1978) : Le moi dans la théorie de Freud et dans la technique de la psychanalyse, Le Séminaire, livre II (1955-1956). Paris, Seuil.

LACAN, Jacques (2004) : Le Séminaire, livre IX, L'angoisse (1962-1963). Paris, Le Seuil. 
LagorgetTe, Dominique (1998): Désignatifs et termes d'adresse dans quelques texte. en moyen français. Thèse de doctorat sous la direction de Michèle Perret. Paris, Université de Paris X Nanterre.

LAGORGETTE, Dominique (2003) : «Termes d'adresse, insulte et notion de détachement en diachronie : quels critères d'analyse pour la fonction d'adresse? ". Cahiers de praxématique, 40, 43-70.

LAGORGETTE, Dominique (2007) : «Termes d'adresse et insultes : discours sur l'autre et sur moi? ", in W. Ayres-Bennett et M.C. Jones (éds.), The French Language and questions of identity, Londres, MHRA / Legenda, 116-128.

LagorgetTe, Dominique et Pierre LARRIVÉE [dir.] (2004) : Les insultes : approches sémantiques et pragmatiques, in Langue française 144 [numéro spécial].

LALANDE, André (1999): Vocabulaire technique et critique de la philosophie. Paris, Quadrige/PUF, vol. I.

LEHMANN, Sabine (2010) : "L'évolution des termes d'adresse à contenu social en ancien et en moyen français ", Corela, hors-série 8, 1-21. Disponible sur: https://journals.openedition.org/corela/1610.

MiANO, Léonora (2005a) : L'Intérieur de la nuit. Paris, Plon.

MiANO, Léonora (2005b) : "L'intérieur de la nuit, un roman de Léonora Miano. Une traversée des profondeurs de l'Afrique ». Les interviews d'Amina. Lires les femmes écrivains et les littératures africaines, 6427, Entretien par NB. Novembre, 68-69. Disponible sur : http://aflit.arts.uwa.edu.au/AMINAMiano.html; 16/9/2018].

MiANO, Léonora (2006) : Contours du jour qui vient. Paris, Plon (coll. Pocket).

MiANO, Léonora (2009) : Les aubes écarlates. Paris, Plon.

MiANO, Léonora (2016) : Crépuscule du tourment. Paris, Grasset.

MONTE, Michèle (2005) : « Ô + SN dans les textes poétiques : entre apostrophe et exclamation ». Modèles linguistiques, XXVI (2), 45-68.

MONTE, Michèle (2008) : «Usages littéraires de l'apostrophe : fonctions textuelles et pragmatiques et spécificités génériques ", in Actes du $1^{\text {er }}$ congrès mondial de linguistique française, Paris, EDP Sciences, 1417-1428 [CD-Rom]. Disponible sur: https://www.linguistiquefrancaise.org/articles/cmlf/pdf/2008/01/cmlf08073.pdf.

OpPEnHEIMER, Agnès (2002) : "Identité ", in Alain De Mijolla (dir.), Dictionnaire international de la psychanalyse, Paris, Calmann Lévy, vol. 1, 783-784.

PERRET, Delphine (1968) : "Termes d'adresse et injures. À propos d'un dictionnaire des injures ", Cahiers de lexicologie, 12 (1), 3-14.

PERRET, Delphine (1970) : «Les appellatifs : analyse lexicale et actes de parole », Langages, $32,112-118$.

REY-DEBOVE, Josette (1997) : Le métalangage. Étude linguistique du discours sur le langage. Paris, Armand Colin/Masson.

Rodríguez Somolinos, Amalia (2016) : «Présentation », Linx, 73 (A. Rodríguez Somoli- 
nos, dir., Énonciation et marques d'oralité dans l'évolution du français). Disponible sur : http://journals.openedition.org/linx/1656.

TAYLOR, Charles (1998 [1979]) : Hegel et la société moderne. Québec/Paris, Les presses de l'Université Laval/Le Cerf.

VIVES, Jean-Michel (1999-2000): "À l'entour de la pulsion invocante", in Séminaire de psychanalyse, 143-150. Disponible sur : http://www.gnipl.fr/pdf_actes_sem5/A\%201\%27entour\%20de\%20la\%20pulsion\%20invocante\%20Jean-Michel\%20Vives.pdf.

YANGE, Paul (2006) : "Leonora Miano : l'Afrique a besoin d'entendre un discours de responsabilité, même si cela déplaît ». Entretien sur Grioo.com, 23 mai. Disponible sur : https://www.grioo.com/info6910.html.

Para citar este artículo / Pour citer cet article :

PeÑalver ViCEA, Maribel (2019): «Termes d'adresse et pulsion invocante chez Léonora Miano». Çédille. revista de estudios franceses, 16, 381-406. DOI: https://doi.org/10.25145/j.cedille.2019.17.16.22. 